Алгебра и анализ

Том 21 (2009), № 5
St. Petersburg Math. J. Vol. 21 (2010), No. 5, Pages 693-704 S 1061-0022(2010)01112-9

Article electronically published on July 14, 2010

\title{
ON THE SINGULAR BRAID MONOID
}

\author{
V. VERSHININ
}

\begin{abstract}
Garside's results and the existence of the greedy normal form for braids are shown to be true for the singular braid monoid. An analog of the presentation of J. S. Birman, K. H. Ko, and S. J. Lee for the classical braid group is also obtained for this monoid.
\end{abstract}

\section{§1. INTRODUCTION}

Various questions concerning braid groups and their generalizations have attracted attention during the last decades. Presentations of braid groups and algorithmic problems for the singular braid monoid are among these questions.

The canonical presentation of the braid group on $n$ strings $B r_{n}$ was given by Artin [5] and is well known. It has the generators $\sigma_{1}, \sigma_{2}, \ldots, \sigma_{n-1}$ and the relations

$$
\begin{cases}\sigma_{i} \sigma_{j}=\sigma_{j} \sigma_{i} & \text { if }|i-j|>1, i, j=1, \ldots, n-1 ; \\ \sigma_{i} \sigma_{i+1} \sigma_{i}=\sigma_{i+1} \sigma_{i} \sigma_{i+1}, & i=1, \ldots, n-2 .\end{cases}
$$

Of course, there exist other presentations of the braid group. Birman, Ko and Lee [8] introduced a presentation with generators $a_{t s}$ with $1 \leq s<t \leq n$, and the relations

$$
\begin{cases}a_{t s} a_{r q}=a_{r q} a_{t s} & \text { for } \quad(t-r)(t-q)(s-r)(s-q)>0, \\ a_{t s} a_{s r}=a_{t r} a_{t s}=a_{s r} a_{t r} & \text { for } 1 \leq r<s<t \leq n .\end{cases}
$$

The generators $a_{t s}$ are expressed in terms of the canonical generators $\sigma_{i}$ as follows:

$$
a_{t s}=\left(\sigma_{t-1} \sigma_{t-2} \cdots \sigma_{s+1}\right) \sigma_{s}\left(\sigma_{s+1}^{-1} \cdots \sigma_{t-2}^{-1} \sigma_{t-1}^{-1}\right) \quad \text { for } \quad 1 \leq s<t \leq n
$$

The singular braid monoid $S B_{n}$ or Baez-Birman monoid [6, 7] is defined as a monoid with generators $\sigma_{i}, \sigma_{i}^{-1}, x_{i}, i=1, \ldots, n-1$, and the relations

$$
\begin{cases}\sigma_{i} \sigma_{j}=\sigma_{j} \sigma_{i} & \text { if }|i-j|>1, \\ x_{i} x_{j}=x_{j} x_{i} & \text { if }|i-j|>1, \\ x_{i} \sigma_{j}=\sigma_{j} x_{i} & \text { if }|i-j| \neq 1, \\ \sigma_{i} \sigma_{i+1} \sigma_{i}=\sigma_{i+1} \sigma_{i} \sigma_{i+1}, & \\ \sigma_{i} \sigma_{i+1} x_{i}=x_{i+1} \sigma_{i} \sigma_{i+1}, & \\ \sigma_{i+1} \sigma_{i} x_{i+1}=x_{i} \sigma_{i+1} \sigma_{i}, & \\ \sigma_{i} \sigma_{i}^{-1}=\sigma_{i}^{-1} \sigma_{i}=1 . & \end{cases}
$$

In pictures, $\sigma_{i}$ corresponds to the canonical generator of the braid group and $x_{i}$ represents the intersection of the $i$ th and $(i+1)$ st strands as in Figure1. The basic motivation for the introduction of this object was the Vassiliev-Goussarov theory of finite type invariants.

2010 Mathematics Subject Classification. Primary 20F36; Secondary 20 F38.

Key words and phrases. Braid group, singular braid monoid, word problem, Garside normal form. 

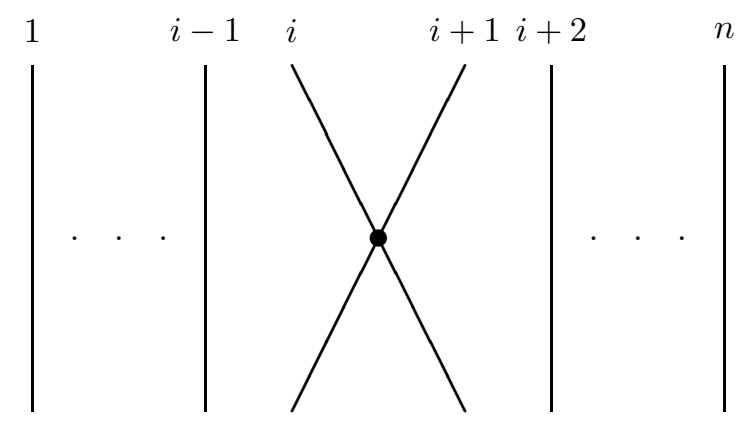

FiguRE 1

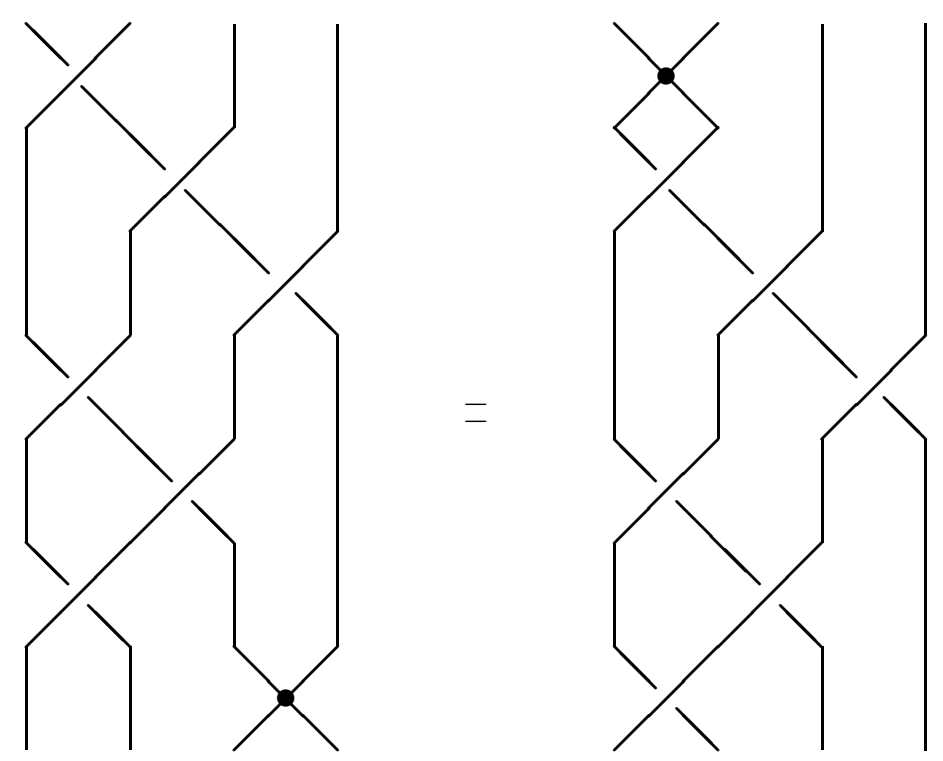

FigURE 2

The singular braid monoid on two strings is isomorphic to $\mathbb{Z} \oplus \mathbb{Z}^{+}$, so that the word problem in this case is trivial. For the monoid with three strings this problem was solved by Járai [16] and Dasbach and Gemein [11. In the general case, this was done by Corran in the complicated and technical paper [10. Here we hope to give a simple solution using almost nothing but F. A. Garside's arguments. The initial idea is simple and geometric: the behavior of the Garside fundamental element determined by the word $\Delta$ (for convenience, the definition is given below in formula (4)) with respect to the singular generators $x_{i}$ is similar to that with respect to the braid generators $\sigma_{i}$ :

$$
x_{i} \Delta \doteq \Delta x_{n-i},
$$

as shown in Figure 2

\section{§2. THE WORD PROBLEM FOR THE SINGULAR BRAID MONOID}

Following Garside's ideas, we consider the positive singular braid monoid $S B_{n}^{+}$. It is defined as a monoid with the generators $\sigma_{i}, x_{i}, i=1, \ldots, n-1$, and relations (3) except the last one, which concerns the invertibility of $\sigma_{i}$. We denote by $\mathcal{A}$ the alphabet $\left\{\sigma_{i}, x_{i}\right.$, $(i=1, \ldots, n-1)\}$. We say that two positive words $A$ and $B$ in the alphabet $\mathcal{A}$ are 
positively equal if they are equal as elements of $S B_{n}^{+}$. In this case we write $A \doteq B$. The identity of words is denoted by the symbol $\equiv$. We denote by $L(W)$ the word-length of the word $W$. As usual, we say that an element $a$ in a monoid is a (left) divisor of an element $b$ if there exists an element $c$ such that $a c=b$ in the monoid; in this case we say that $b$ is a (right) multiple of $a$. The relation of divisibility is a partial order in $S B_{n}^{+}$, and the notions of the greatest common divisor and the least common multiple make sense.

The proofs of the statements below are the same as in Garside's paper [15] with some exceptions as, for example, the proof of Proposition 6, where we use the Mal'tsev rule. Proposition 6 was proved by Corran [10; Theorem 3 was proved by Fenn, Rolfsen and Zhu [14] by different methods.

Proposition 1. The following claims are true in $S B_{n}^{+}$for $i, k=1, \ldots, n-1$. Given $\sigma_{i} A \doteq \sigma_{k} B$, where $A$ and $B$ are (positive) words in the alphabet $\mathcal{A}$, we have:

$$
\begin{array}{ll}
\text { if } k=i, & \text { then } A \doteq B, \\
\text { if }|k-i|=1, & \text { then } A \doteq \sigma_{k} \sigma_{i} Z, B \doteq \sigma_{i} \sigma_{k} Z \text { for some word } Z, \\
\text { if }|k-i| \geq 2, & \text { then } A \doteq \sigma_{k} Z, B \doteq \sigma_{i} Z \text { for some word } Z ;
\end{array}
$$

given $\sigma_{i} A \doteq x_{k} B$, we have:

$$
\begin{array}{ll}
\text { if }|k-i|=1, \quad \text { then } & A \doteq \sigma_{k} x_{i} Z, B \doteq \sigma_{i} \sigma_{k} Z \text { for some } Z \text {, } \\
\text { if } \quad|k-i| \neq 1, & \text { then } A \doteq x_{k} Z, B \doteq \sigma_{i} Z \text { for some } Z ;
\end{array}
$$

and given $x_{i} A \doteq x_{k} B$, we have:

$$
\begin{array}{ll}
\text { if } k=i, & \text { then } A \doteq B, \\
\text { if }|k-i| \geq 2, & \text { then } A \doteq x_{k} Z, B \doteq x_{i} Z \text { for some } Z, \\
\text { the case where }|k-i|=1 & \text { is impossible. }
\end{array}
$$

The same is true for the right multiples of $\sigma_{i}$ or $x_{k}$.

Proof. Garside's proof works here. We apply induction on the length $s$ of the word $A$ and the length of the chain of transformations from $a_{i} A$ to $a_{k} B$, where $a_{i}$ may be $\sigma_{i}$ or $x_{i}$, and $a_{k}$ may be $\sigma_{k}$ or $x_{k}$. The cases of $s=0,1$ are obvious; so, suppose that the statement is true for $s \leq r$ and that for $s=r+1$ it is true if the chain-length does not exceed $t$. As an example, we prove the last statement, which is formally not contained in Garside's considerations. So, let $A, B$ be of word-length $r+1$ and let $x_{i} A \doteq x_{k} B$, $|i-k|=1$, through a transformation of chain-length $t+1$. We may assume that $k=i+1$ and let the successive words of the transformations be

$$
W_{1} \equiv x_{i} A, \ldots, W_{t+2} \equiv x_{i+1} B .
$$

We choose an arbitrary intermediate word $W_{g}$, say, somewhere in the middle of the chain. We have $W_{g} \equiv a V$, where $a$ is a generator of $S B_{n}^{+}$. First, suppose that $a$ commutes with $x_{i}, x_{i+1}$; then $x_{i} A \doteq a V \doteq x_{i+1} B$ and by induction we obtain

$$
A \doteq a P, \quad V \doteq x_{i} P, \quad V \doteq x_{i+1} Q, \quad B \doteq a Q .
$$

Thus, $x_{i} P \doteq x_{i+1} Q$, which is impossible by induction.

The cases where $a=x_{i-1}, x_{i}, x_{i+1}, x_{i+2}$ are also impossible by induction. The cases that need consideration are $a=\sigma_{i-1}, \sigma_{i}, \sigma_{i+1}, \sigma_{i+2}$. So, let $a=\sigma_{i-1}$; then $W_{g} \doteq \sigma_{i-1} V$. By induction we get

$$
A \doteq \sigma_{i-1} \sigma_{i} P, \quad V \doteq \sigma_{i} x_{i-1} P, \quad V \doteq x_{i+1} Q, \quad B \doteq \sigma_{i-1} Q .
$$

Hence, $\sigma_{i} x_{i-1} P \doteq x_{i+1} Q$, whence $x_{i-1} P \doteq \sigma_{i+1} x_{i} R, Q \doteq \sigma_{i} \sigma_{i+1} R$. Again by induction we get $P \doteq \sigma_{i+1} S, x_{i} R \doteq x_{i-1} S$, which is impossible. The remaining cases may be analyzed in the same way. 
Corollary 1. If $A \doteq P, B \doteq Q, A X B \doteq P Y Q(L(A) \geq 0, L(B) \geq 0)$, then $X \doteq Y$. That is, the monoid $S B_{n}^{+}$is left and right cancellative.

Proposition 2. For any word $W$ in the alphabet $\mathcal{A}=\left\{\sigma_{i}, x_{i}(i=1, \ldots, n-1)\right\}$, let $S$ be a word in the alphabet $\mathcal{S}=\left\{\sigma_{i}(i=1, \ldots, n-1)\right\}$ of maximal length such that $W \doteq S T$ for some word $T$. Suppose also that $W \doteq A V$ for some word $A$ in the alphabet $\left\{\sigma_{i}\right\}$. Then $S$ is divisible by $A$. The same is true for right division.

Proof. We use induction on the length of $S$. If $S$ has length 1, then $A$ also has length 1 and the assertion follows from Proposition 1. Suppose the claim is true if the length is less than or equal to $k$, and let the length of $S$ be equal to $k+1$. First, we consider the case where the length of $A$ is equal to 1 . This means that $A \equiv \sigma_{j}$. Let the first letter of $S$ be $\sigma_{i}: S \equiv \sigma_{i} S^{\prime}$, so that we have the situation $\sigma_{i} S^{\prime} T \doteq \sigma_{j} V$. If $i=j$, then we are done. If $|i-j| \geq 2$, then $S^{\prime} T \doteq \sigma_{j} X$, and induction yields $S^{\prime} \doteq \sigma_{j} R^{\prime}$. If $|i-j|=1$, then $S^{\prime} T \doteq \sigma_{j} \sigma_{i} Y$, and again by induction we obtain $S^{\prime} \doteq \sigma_{j} \sigma_{i} Q^{\prime}$. Suppose now that the length of $A$ is greater than l; then $A \equiv \sigma_{j} A^{\prime}$ for some $\sigma_{j}$. Using the preceding case, we have $S \doteq \sigma_{j} S^{\prime}$ and $S^{\prime} T \doteq A^{\prime} V$, and induction shows that $S^{\prime} \doteq A^{\prime} S^{\prime \prime}$. Hence, $S$ is divisible by $A$.

Corollary 2. For any element $w$ of $S B_{n}^{+}$there exists a unique greatest left (right) divisor that belongs to $\mathrm{Br}_{n}^{+}$.

Garside's fundamental element in the braid group $B r_{n}$ is defined by the following word:

$$
\Delta \equiv \sigma_{1} \ldots \sigma_{n-1} \sigma_{1} \ldots \sigma_{n-2} \ldots \sigma_{1} \sigma_{2} \sigma_{1} .
$$

If we use Garside's notation $\Pi_{t} \equiv \sigma_{1} \ldots \sigma_{t}$, then $\Delta \equiv \Pi_{n-1} \ldots \Pi_{1}$. We keep the same notation for the corresponding images in $S B_{n}$. Garside's transformation of words $\mathcal{R}$ is defined by the formula

$$
\mathcal{R}\left(\sigma_{i}\right) \equiv \sigma_{n-i} .
$$

This gives an automorphism of $B r_{n}$ and the positive braid monoid $\mathrm{Br}_{n}^{+}$. We extend it to letters $x_{i}$ and, thus, to $S B_{n}^{+}$and to $S B_{n}$ by

$$
\mathcal{R}\left(x_{i}\right) \equiv x_{n-i} .
$$

Proposition 3. In $S B_{n}^{+}$we have

$$
\begin{aligned}
\sigma_{i} \Delta & \doteq \Delta \mathcal{R}\left(\sigma_{i}\right), \\
x_{i} \Delta & \doteq \Delta \mathcal{R}\left(x_{i}\right) .
\end{aligned}
$$

Proof. For $i=1$, we have

$$
\begin{aligned}
x_{1} \Delta & \equiv x_{1} \sigma_{1} \ldots \sigma_{n-1} \sigma_{1} \ldots \sigma_{n-2} \ldots \sigma_{1} \sigma_{2} \sigma_{1} \doteq \sigma_{1} x_{1} \sigma_{2} \ldots \sigma_{n-1} \sigma_{1} \ldots \sigma_{n-2} \ldots \sigma_{1} \sigma_{2} \sigma_{1} \\
& \doteq \sigma_{1} x_{1} \sigma_{2} \sigma_{1} \ldots \sigma_{n-1} \sigma_{2} \ldots \sigma_{n-2} \ldots \sigma_{1} \sigma_{2} \sigma_{1} \\
& \doteq \sigma_{1} \sigma_{2} \sigma_{1} x_{2} \sigma_{3} \ldots \sigma_{n-1} \sigma_{2} \ldots \sigma_{n-2} \ldots \sigma_{1} \sigma_{2} \sigma_{1} \\
& \doteq \sigma_{1} \sigma_{2} \sigma_{1} \sigma_{3} \ldots x_{n-2} \sigma_{n-1} \sigma_{n-2} \ldots \sigma_{1} \sigma_{2} \sigma_{1} \\
& \doteq \sigma_{1} \sigma_{2} \sigma_{1} \sigma_{3} \ldots \sigma_{n-2} \sigma_{n-1} x_{n-1} \ldots \sigma_{1} \sigma_{2} \sigma_{1} \\
& \doteq \sigma_{1} \sigma_{2} \sigma_{3} \ldots \sigma_{n-2} \sigma_{n-1} \sigma_{1} \ldots \sigma_{n-2} \ldots \sigma_{1} \sigma_{2} \sigma_{1} x_{n-1} \equiv \Delta x_{n-1} .
\end{aligned}
$$


For $i \geq 2$, we have

$$
\begin{aligned}
x_{i} \Delta & \equiv x_{i} \sigma_{1} \ldots \sigma_{n-1} \sigma_{1} \ldots \sigma_{n-2} \ldots \sigma_{1} \sigma_{2} \sigma_{1} \\
& \doteq \sigma_{1} \ldots x_{i} \sigma_{i-1} \sigma_{i} \ldots \sigma_{n-1} \sigma_{1} \ldots \sigma_{n-2} \ldots \sigma_{1} \sigma_{2} \sigma_{1} \\
& \doteq \sigma_{1} \ldots \sigma_{i-1} \sigma_{i} x_{i-1} \ldots \sigma_{n-1} \sigma_{1} \ldots \sigma_{n-2} \ldots \sigma_{1} \sigma_{2} \sigma_{1} \doteq \Pi_{n-1} x_{i-1} \Pi_{n-2} \ldots \Pi_{1} \\
& \doteq \Pi_{n-1} \ldots \Pi_{n-i+1} x_{1} \Pi_{n-i} \Pi_{n-i-1} \ldots \Pi_{1} \\
& \doteq \Pi_{n-1} \ldots \Pi_{n-i+1} \Pi_{n-i} \Pi_{n-i-1} x_{n-i} \Pi_{n-i-2} \ldots \Pi_{1} \\
& \doteq \Pi_{n-1} \ldots \Pi_{n-i+1} \Pi_{n-i} \ldots \Pi_{1} x_{n-i} \doteq \Delta x_{n-i} .
\end{aligned}
$$

The fundamental element has remarkable divisibility properties.

Proposition 4 ([15], Lemma 4). In Br $r_{n}$, there exist positive words $W_{r}$ and $V_{r}$ such that

$$
\Delta \doteq \sigma_{r} W_{r} \doteq V_{r} \sigma_{r}, \quad r=1, \ldots, n-1 .
$$

Proposition 5. If $W$ is an arbitrary positive word in the alphabet $\mathcal{A}$ such that either

$$
W \doteq \sigma_{1} A_{1} \doteq \sigma_{2} A_{2} \doteq \cdots \doteq \sigma_{n-1} A_{n-1},
$$

or

$$
W \doteq B_{1} \sigma_{1} \doteq B_{2} \sigma_{2} \doteq \cdots \doteq B_{n-1} \sigma_{n-1}
$$

in $S B_{n}^{+}$, then $W \doteq \Delta Z$ for some $Z$.

In the proof of the next proposition, we use the Mal'tsev rule [2]-4]. Originally, it was formulated for groups. Here we need a version for monoids; the proof is the same. We consider monoids some generators of which are invertible. All relations except invertibility (such as the last relation in (3D) will be called principal. In the Mal'tsev rule the following situation is considered: two words $A$ and $C$, where $C$ contain no negative degrees of letters of the alphabet, represent one and the same element of the monoid. Then there exists a sequence of words

$$
A \equiv A_{0} \rightarrow A_{1} \rightarrow \cdots \rightarrow A_{j} \rightarrow \cdots \rightarrow A_{k} \equiv C,
$$

where each arrow means an elementary operation, which may be an application of one principal defining relation or insertion or deletion of an expression $c c^{-1}$ or $c^{-1} c$, where $c$ is a letter of our alphabet. In the first case, insertion is said to be left and in the second it is right. A right insertion

$$
A_{j} \equiv Y_{j} Z_{j} \rightarrow Y_{j} c^{-1} c Z_{j}
$$

is correct if the fragment $Y_{j}$ is not changed in the sequence of transformations (5) till the elimination of $c^{-1}$. For left insertion the same condition should be fulfilled for the fragment $Z_{j}$. We use the Mal'tsev rule in the following form.

If all principal defining relations of a monoid contain only positive degrees of letters of the alphabet, then the transformation of a word $A$ into a positive word $C$ can be done by a sequence of operations whose insertions are all correct.

Proposition 6. The canonical homomorphism

$$
S B_{n}^{+} \rightarrow S B_{n}
$$

is a monomorphism.

Proof. We need to prove that if two elements of $S B_{n}^{+}$(expressed by positive words $A$ and $C$ ) are equal in $S B_{n}$, then they are positively equal. If the elements determined by the words $A$ and $C$ are equal in $S B_{n}$, then there exists a sequence of words (5) where in any insertion or deletion of the expression $c c^{-1}$ or $c^{-1} c$ the generator $c$ is one of the $\sigma_{i}{ }^{\text {'s. }}$. 
Consider a subsequence of (5) that starts with the last (e.g., right) insertion and finishes with the deletion of the corresponding $c^{-1}$ :

$$
A_{j} \equiv Y_{j} Z_{j} \rightarrow Y_{j} c^{-1} c Z_{j} \rightarrow Y_{j} c^{-1} V_{j+1} \rightarrow \cdots \rightarrow Y_{j} c^{-1} c Z_{j+r} \rightarrow Y_{j} Z_{j+r}
$$

The fragment $Y_{j}$ does not change in this subsequence because the insertion is correct. For any $c$ there exists a positive word $D_{c}$ such that $D_{c} c \doteq \Delta$. Define the sequence

$$
\begin{aligned}
\Delta A_{j} & \equiv \Delta Y_{j} Z_{j} \mapsto \cdots \mapsto \mathcal{R}\left(Y_{j}\right) \Delta Z_{j} \mapsto \cdots \mapsto \mathcal{R}\left(Y_{j}\right) D_{c} c Z_{j} \hookrightarrow \mathcal{R}\left(Y_{j}\right) D_{c} V_{j+1} \\
& \hookrightarrow \cdots \hookrightarrow \mathcal{R}\left(Y_{j}\right) D_{c} c Z_{j+r} \mapsto \cdots \mapsto \mathcal{R}\left(Y_{j}\right) \Delta Z_{j+r} \mapsto \cdots \mapsto \Delta Y_{j} Z_{j+r},
\end{aligned}
$$

where $\mapsto$ denotes a positive operation and $\hookrightarrow$ denotes a positive operation or deletion. Thus, multiplying by $\Delta$ we eliminate one insertion. By induction, we construct a sequence of positive operations between $\Delta^{m} A \Delta^{l}$ and $\Delta^{m} C \Delta^{l}$ for some $m$ and $l$. After cancellation, this means that $A$ and $C$ are positive equivalent. As was noted by V. V. Chaynikov, one can get a proof without invoking the Mal'tsev rule but using the fact that relations (3) are invariant with respect to the operation $\mathcal{R}$.

We introduce the lexicographical ordering among all positive words in the alphabet $\mathcal{A}=\left\{\sigma_{1}, \ldots, \sigma_{n-1}, x_{1}, \ldots, x_{n-1}\right\}$, assuming that $\sigma_{1}<\sigma_{2}<\cdots<\sigma_{n-1}<x_{1}<x_{2}<$ $\ldots<x_{n-1}$. The base of a positive word $W$ is the smallest positive word with respect to this ordering that is positively equal to $W$. The base is uniquely determined. If for a positive word $A$ the element in $S B_{n}^{+}$that represents $A$ is not a multiple of $\Delta$, then we write $\bar{A}$ for the base of $A$.

Theorem 1. Every element $w$ in $S B_{n}$ can be expressed uniquely in the form $\Delta^{m} \bar{A}$, where $m$ is an integer.

Proof. First, suppose that an element $w$ is represented by a positive word $P$. Among all positive words positively equivalent to $P$, we choose a word in the form $\Delta^{t} A$ with $t$ maximal. Then $A$ is not a multiple of $\Delta$, and we have

$$
P \doteq \Delta^{t} \bar{A} \text {. }
$$

Now, let $W$ be an arbitrary word in $S B_{n}$. Then we can put

$$
W \equiv W_{1}\left(c_{1}\right)^{-1} W_{2}\left(c_{2}\right)^{-1} \ldots\left(c_{k}\right)^{-1} W_{k+1},
$$

where each $W_{j}$ is a positive word of nonnegative length and the $c_{l}$ are generators $\sigma_{i}$, the only possible invertible generators. As has already been mentioned, for each $c_{l}$ there exists a positive word $D_{l}$ such that $c_{l} D_{l} \doteq \Delta$, so that $\left(c_{l}\right)^{-1}=D_{l} \Delta^{-1}$, whence

$$
W=W_{1} D_{1} \Delta^{-1} W_{2} D_{2} \Delta^{-1} \ldots W_{k} D_{k} \Delta^{-1} W_{k+1} .
$$

Hence, moving the factors $\Delta^{-1}$ to the left, we obtain $W=\Delta^{k} P$, where $P$ is positive, and expressing this in the form $\Delta^{t} \bar{A}$, finally we get

$$
W=\Delta^{m} \bar{A} \text {. }
$$

It remains to show that the form (6) is unique. Suppose

$$
\Delta^{m} \bar{A}=\Delta^{p} \bar{C} \text {. }
$$

Let $p<m$, and let $m-p=t>0$. Then (7) gives $\Delta^{t} \bar{A}=\bar{C}$, which is impossible. Consequently, $p=m$, whence $\bar{A}=\bar{B}$. Thus, by Proposition 6 , we obtain $\bar{A} \doteq \bar{C}$, but the base is unique, so that $\bar{A} \equiv \bar{C}$ and the uniqueness of the form (6) is established.

The form of an element $w$ established in this theorem is called the Garside left normal form, and the index $m$ is called the power of $w$. The Garside right normal form is defined similarly, and the corresponding version of Theorem 1 is true. The Garside normal form also gives a solution to the word problem in the singular braid monoid. 
Corollary 3. Two words in the alphabet $\mathcal{A}$ represent one and the same element in $S B_{n}$ if and only if their Garside normal forms (left or right) are identical.

The Garside normal form for the braid groups was refined in the subsequent papers [1, 13, 12]. Namely, the left-greedy form (in the terminology of W. Thurston [13])

$$
\Delta^{t} A_{1} \ldots A_{k}
$$

was introduced, where the $A_{i}$ are the successive longest possible fragments of the word $\Delta$ (in the terminology of Adyan [1]), or positive permutation braids (in the terminology of El-Rifai and Morton [12]). Certainly, the right-greedy form is defined similarly.

We consider these forms for the singular braid monoid. First, for any word $W$ we move the greatest power of $\Delta$ to the left. To the right we have a positive word $W^{\prime}$ not divisible by $\Delta$. Consider the decomposition $W^{\prime} \doteq S_{1} T$ as in Proposition 2 , Then we take the fragments of the left-greedy form for $S_{1}: S_{1,1} \ldots S_{1, t}$. Among all the $x_{i}$-divisors of $T$, we choose the smallest in the lexicographical order: $T \doteq x_{i_{1}} T_{1}$. Consider the decomposition as in Proposition 2 for $T_{1}$ and continue this process. We get the form

$$
W \doteq \Delta^{t} S_{1} X_{1} \ldots S_{k} X_{k},
$$

where each $S_{i}$ consists of fragments of the left-greedy form for the braid group and each $X_{i}$ is a lexicographically ordered product of $x_{j}$. We call this form the left-greedy form for the singular braid monoid. The right-greedy form for the singular braid monoid is defined in the same way.

Theorem 2. Each element of the singular braid monoid can be written uniquely in a left-greedy (right-greedy) form.

Theorem 3. For $n=2$ the singular braid monoid $S B_{n}$ is commutative and isomorphic to $\mathbb{Z} \oplus \mathbb{Z}^{+}$. For $n \geq 3$, the center of $S B_{n}$ is the same as the center of $B r_{n}$ and, thus, it is generated by $\Delta^{2}$.

\section{§3. Conjugacy Problem for the Singular Braid MONoid}

Let $M$ be a monoid with unit group $G$. We say that two elements $u, v \in M$ are conjugate if $v=g^{-1} u g$ for some $g \in G$. This means that conjugate elements $u$ and $v$ in $S B_{n}$ belong to one and the same orbit of the canonical action of the braid group $B r_{n}$ on $S B_{n}$, and the conjugacy problem for the singular braid monoid will be understood precisely in this sense. Such an approach to conjugacy appears among others in monoid theory; see, e.g., [17.

Since the relations (3) are homogeneous with respect to both types of generators $\sigma_{i}$ and $x_{i}$, the following three homomorphisms, which express the degree of an element with respect to $\sigma_{i}, x_{i}$ and the total degree, are well defined: $\operatorname{deg}_{\sigma}: S B_{n} \rightarrow \mathbb{Z}, \operatorname{deg}_{x}: S B_{n} \rightarrow$ $\mathbb{Z}^{+}$, and $\operatorname{deg}: S B_{n} \rightarrow \mathbb{Z}$.

Proposition 7. The group of units of the monoid $S B_{n}$ is equal to the image of the braid group $\mathrm{Br}_{n}$.

Proof. Since invertible elements must have invertible degree $\operatorname{deg}_{x}$, we have $\operatorname{deg}_{x}=0$.

Garside's solution of the conjugacy problem for braid groups works in the case of the singular braid monoid. In the proof of the following theorem concerning the structure of the Cayley diagram $D(W)$ of a positive word $W$ in the alphabet $\mathcal{A}=\left\{\sigma_{i}, x_{k}\right\}$ containing $\Delta$, some additional considerations are necessary.

Theorem 4. If $W \doteq \Delta V$ is any positive word (in the alphabet $\mathcal{A}$ ) containing $\Delta$, then each node of $D(W)$ is incident with each edge $\sigma_{i}, i=1, \ldots, n-1$. 


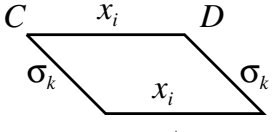

a)

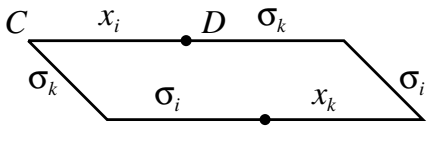

b)

Figure 3

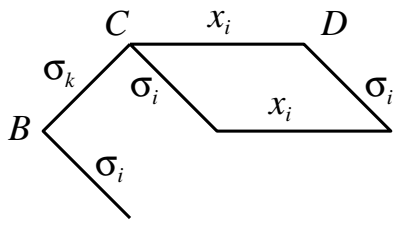

a)

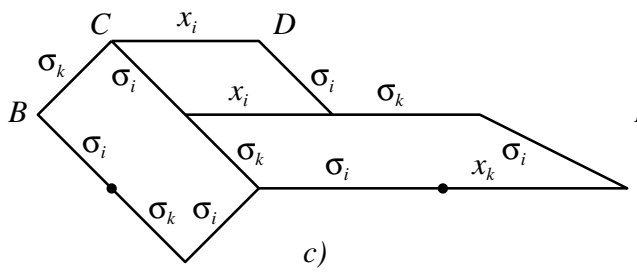

c)

FiguRE 4
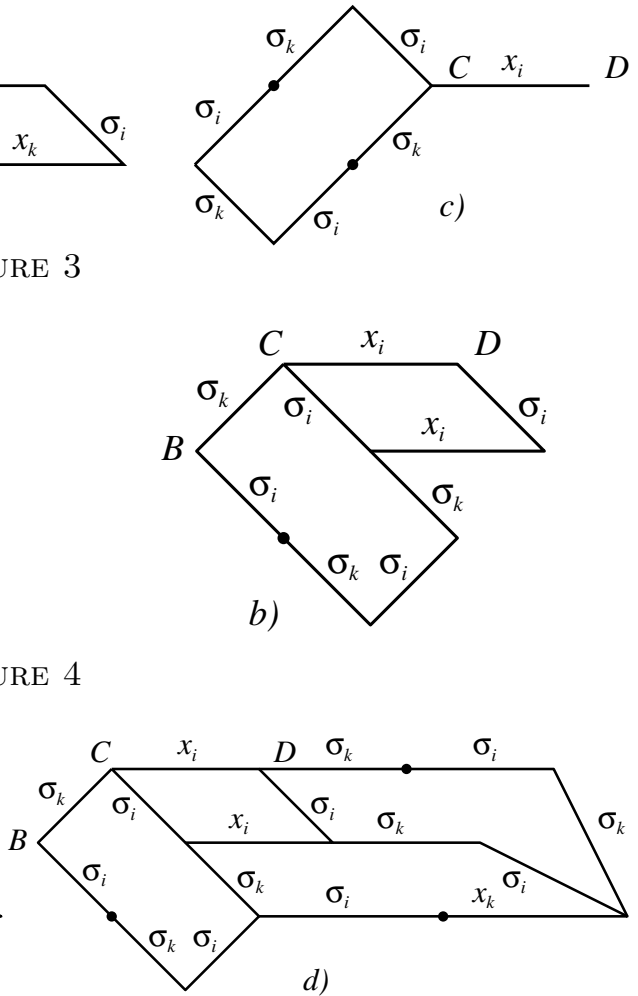

Figure 5

Proof. We follow Garside's proof, which employs induction on the order of a node equal to the word-length of an element. Proposition 4 gives the beginning of this induction: a statement for the identity element.

Assume that the statement of the theorem is true for all nodes of degree not exceeding $m$. Let $C$ be an arbitrary node of order $m$, and let an edge $\sigma_{i}$ start at $C$ and end at a node $D$ of order $m+1$, the case analyzed by Garside. By the inductive hypothesis, the node $C$ is also incident to all $\sigma_{k}, k \neq i$. Then the braid relations and Proposition 1 show that the node $D$ is also incident to all $\sigma_{k}, k \neq i$.

Now we consider the case of an edge $x_{i}$ that starts at some node $C$ of order $m$ and ends at a node $D$ of order $m+1$. By the inductive hypothesis, the node $C$ is also incident to all $\sigma_{k}, k \neq i$. First, we treat the case of the generators $\sigma_{k}$ with $|k-i| \neq 1$. If the edge $\sigma_{k}$ ends at $C$, then $\sigma_{k} x_{i} \doteq x_{i} \sigma_{k}$, which means that the edge $\sigma_{k}$ also ends at $D$. If $\sigma_{k}$ starts at $C$, then we can use Proposition 1 to obtain the fragment of the Cayley graph depicted in Figure 3 a). Let $|k-i|=1$. If the edge $\sigma_{k}$ starts at $C$, then Proposition 1 yields the fragment of the Cayley graph depicted in Figure $3 \mathrm{~b}$ ). Now, consider the case where the edge $\sigma_{k}$ ends at $C$ (and starts at some node $B$ ). The edge $\sigma_{i}$ must be incident to $C$, so that it either ends or starts at $C$. In the first case, Proposition 1 gives us the fragment of the Cayley graph depicted in Figure $3 \mathrm{c}$ ). We complete the proof in this case by using the relation $\sigma_{i} \sigma_{k} x_{i}=x_{k} \sigma_{i} \sigma_{k}$. In the last case, the edge $\sigma_{i}$ starts at $C$. Consider the node $B$ where $\sigma_{k}$ starts. If the edge $\sigma_{i}$ ends at $B$, then we use the relation $\sigma_{i} \sigma_{k} x_{i}=x_{k} \sigma_{i} \sigma_{k}$ and we are done. So, suppose that the edge $\sigma_{i}$ also starts at $B$. Using Proposition 11, we obtain the fragment of the Cayley graph depicted in Figure 4 a). Using Proposition 1 twice, we obtain the fragment of the Cayley graph depicted in Figure 4 b). Finally, using Proposition 1 once again, we come to the fragment of the Cayley graph depicted 

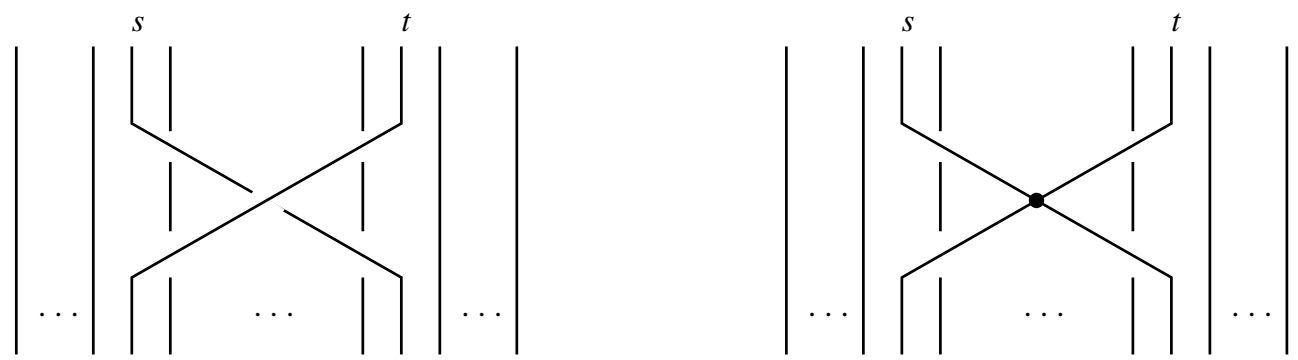

FiguRE 6

in Figure $5 \mathrm{c}$ ). To complete the proof in this case we use the relation $\sigma_{i} \sigma_{k} x_{i}=x_{k} \sigma_{i} \sigma_{k}$, arriving at Figure $5 \mathrm{~d}$ ).

The definition of the summit set for the singular braid monoid is the same as Garside's.

Theorem 5. In $S B_{n}$, two elements are conjugate if and only if their summit sets are identical.

Since for any element of $S B_{n}$ the summit set is finite and is obtained algorithmically, this theorem gives a solution of the conjugacy problem.

\section{§4. Birman-Ko-LeE PRESEntation For the Singular BRAid MONOID}

For the singular braid monoid, we prove the existence of an analog of the presentation (2) of J. S. Birman, K. H. Ko, and S. J. Lee. For $1 \leq s<t \leq n$ and $1 \leq p<q \leq n$, consider the elements of $S B_{n}$ defined by

$$
\begin{cases}a_{t s}=\left(\sigma_{t-1} \sigma_{t-2} \cdots \sigma_{s+1}\right) \sigma_{s}\left(\sigma_{s+1}^{-1} \cdots \sigma_{t-2}^{-1} \sigma_{t-1}^{-1}\right) & \text { for } 1 \leq s<t \leq n, \\ a_{t s}^{-1}=\left(\sigma_{t-1} \sigma_{t-2} \cdots \sigma_{s+1}\right) \sigma_{s}^{-1}\left(\sigma_{s+1}^{-1} \cdots \sigma_{t-2}^{-1} \sigma_{t-1}^{-1}\right) & \text { for } 1 \leq s<t \leq n, \\ b_{q p}=\left(\sigma_{q-1} \sigma_{q-2} \cdots \sigma_{p+1}\right) x_{p}\left(\sigma_{p+1}^{-1} \cdots \sigma_{q-2}^{-1} \sigma_{q-1}^{-1}\right) & \text { for } 1 \leq p<q \leq n .\end{cases}
$$

Geometrically, the generators $a_{s, t}$ and $b_{s, t}$ are depicted in Figure 6,

Theorem 6. The singular braid monoid $S B_{n}$ has a presentation with generators $a_{t s}$, $a_{t s}^{-1}$ for $1 \leq s<t \leq n$ and $b_{q p}$ for $1 \leq p<q \leq n$ and with the relations

$$
\begin{cases}a_{t s} a_{r q}=a_{r q} a_{t s} & \text { for }(t-r)(t-q)(s-r)(s-q)>0, \\ a_{t s} a_{s r}=a_{t r} a_{t s}=a_{s r} a_{t r} & \text { for } 1 \leq r<s<t \leq n, \\ a_{t s} a_{t s}^{-1}=a_{t s}^{-1} a_{t s}=1 & \text { for } 1 \leq s<t \leq n, \\ a_{t s} b_{r q}=b_{r q} a_{t s} & \text { for }(t-r)(t-q)(s-r)(s-q)>0, \\ a_{t s} b_{t s}=b_{t s} a_{t s} & \text { for } 1 \leq s<t \leq n, \\ a_{t s} b_{s r}=b_{t r} a_{t s} & \text { for } 1 \leq r<s<t \leq n, \\ a_{s r} b_{t r}=b_{t s} a_{s r} & \text { for } 1 \leq r<s<t \leq n, \\ a_{t r} b_{t s}=b_{s r} a_{t r} & \text { for } 1 \leq r<s<t \leq n, \\ b_{t s} b_{r q}=b_{r q} b_{t s} & \text { for }(t-r)(t-q)(s-r)(s-q)>0 .\end{cases}
$$

Proof. We follow the proof of Birman, Ko, and Lee [8]; we begin with the presentation of $S B_{n}$ with the help of the generators $\sigma_{i}, \sigma_{i}^{-1}, x_{i}, i=1, \ldots, n-1$, and the system of relations (31). We add the new generators $a_{t s}, a_{t s}^{-1}$ for $1 \leq s<t \leq n$ and $b_{q p}$ for $1 \leq p<q \leq n$ defined by (8). Since relations (9) are described by isotopies of singular braids, they must be consequences of (3), and we may add them too. 
In the special case where $t=s+1$, relations (8) imply that $a_{(s+1) s}=\sigma_{s}, a_{(s+1) s}^{-1}=\sigma_{s}^{-1}$, $b_{(s+1) s}=x_{s}$; therefore, we may omit the generators $\sigma_{1}, \ldots, \sigma_{n-1}, \sigma_{1}^{-1}, \ldots, \sigma_{n-1}^{-1}$ to obtain a presentation with the generators $a_{t s}, a_{t s}^{-1}, b_{p q}$. Now, the defining relations are (9) and

$$
\begin{aligned}
& a_{(i+1) i} a_{(j+1) j}=a_{(j+1) j} a_{(i+1) i} \text { if }|i-j|>1, \\
& a_{(i+1) i} a_{(i+2)(i+1)} a_{(i+1) i}=a_{(i+2)(i+1)} a_{(i+1) i} a_{(i+2)(i+1)}, \\
& a_{t s}=\left(a_{t(t-1)} a_{(t-1)(t-2)} \cdots a_{(s+2)(s+1)}\right) a_{(s+1) s}\left(a_{(s+2)(s+1)}^{-1} \cdots a_{(t-1)(t-2)}^{-1} a_{t(t-1)}^{-1}\right), \\
& a_{t s}^{-1}=\left(a_{t(t-1)} a_{(t-1)(t-2)} \cdots a_{(s+2)(s+1)}\right) a_{(s+1) s}^{-1}\left(a_{(s+2)(s+1)}^{-1} \cdots a_{(t-1)(t-2)}^{-1} a_{t(t-1)}^{-1}\right), \\
& b_{(i+1) i} b_{(j+1) j}=b_{(j+1)} b_{(i+1) i} \text { if }|i-j|>1, \\
& b_{(i+1) i} a_{(j+1) j}=a_{(j+1) j} b_{(i+1) i} \text { if }|i-j| \neq 1, \\
& a_{(i+1) i} a_{(i+2)(i+1)} b_{(i+1) i}=b_{(i+2)(i+1)} a_{(i+1) i} a_{(i+2)(i+1)}, \\
& a_{(i+2)(i+1)} a_{(i+1) i} b_{(i+2)(i+1)}=b_{(i+1) i} a_{(i+2)(i+1)} a_{(i+1) i}, \quad \\
& b_{q p}=\left(a_{q(q-1)} a_{(q-1)(q-2)} \cdots a_{(p+2)(p+1)}\right) b_{(p+1) p}\left(a_{(p+2)(p+1)}^{-1} \cdots a_{(q-1)(q-2)}^{-1} a_{q(q-1)}^{-1}\right) \\
& \text { for } 1 \leq p<q \leq n .
\end{aligned}
$$

Now we prove that relations (10)-(18) are consequences of (9). Birman, Ko, and Lee proved that relations (10)-(12) are consequences of the first two relations in (9). The proof for (13) is the same as for (12). Relations (14) are special cases of the last relation in (9). Relations (15) are special cases of the fourth and the fifth relations in (9). To deduce (16), we use the sixth relation in (9):

$$
a_{(i+1) i} a_{(i+2)(i+1)} b_{(i+1) i}=a_{(i+1) i} b_{(i+2) i} a_{(i+2)(i+1)},
$$

and then the seventh relation in (9):

$$
a_{(i+1) i} b_{(i+2) i} a_{(i+2)(i+1)}=b_{(i+2)(i+1)} a_{(i+1) i} a_{(i+2)(i+1)} .
$$

To deduce (17), we use the second, eighth, fifth, and again second relations in (9):

$$
\begin{aligned}
a_{(i+2)(i+1)} a_{(i+1) i} b_{(i+2)(i+1)} & =a_{(i+1) i} a_{(i+2) i} b_{(i+2)(i+1)}=a_{(i+1) i} b_{(i+1) i} a_{(i+2) i} \\
& =b_{(i+1) i} a_{(i+1) i} a_{(i+2) i}=b_{(i+1) i} a_{(i+2)(i+1)} a_{(i+1) i} .
\end{aligned}
$$

To eliminate (18), we apply the second relation in (9) to change the middle pair $a_{(p+2)(p+1)} b_{(p+1) p}$ in (18) to $b_{(p+2) p} a_{(p+1) p}$. Then we apply this process to the pair $a_{(p+3)(p+2)} b_{(p+2) p}$. Ultimately, this process will move the original central letter $b_{(p+1) p}$ to the leftmost position, where it becomes $b_{q p}$. Free cancellation eliminates everything to the right of it, and we are done.

Now we consider the positive singular braid monoid with respect to generators $a_{t s}$ and $b_{t, s}$ for $1 \leq s<t \leq n$. Its relations are (9) except the one concerning the invertibility of $a_{t s}$. We say that positive words $A$ and $B$ in the alphabets $a_{t s}$ and $b_{t, s}$ are positively equivalent if they are equal as elements of this monoid. In this case, as in the preceding section, we write $A \doteq B$.

The fundamental element of Birman, Ko, and Lee is given by the fundamental word $\delta$ by the formula

$$
\delta \equiv a_{n(n-1)} a_{(n-1)(n-2)} \ldots a_{21} \equiv \sigma_{n-1} \sigma_{n-2} \ldots \sigma_{2} \sigma_{1} .
$$

For us it is convenient to express its divisibility by any generator $a_{t s}$ (which was proved in [8] ) in the following form.

Proposition 8. The fundamental word $\delta$ is positively equivalent to a word that begins or ends with any given generator $a_{t s}$. The explicit expression for left divisibility is

$$
\delta \doteq a_{t s} a_{n(n-1)} a_{(n-1)(n-2)} \ldots a_{(t+1) s} a_{t(t-1)} \ldots a_{(s+2)(s+1)} a_{s(s-1)} \ldots a_{21} .
$$



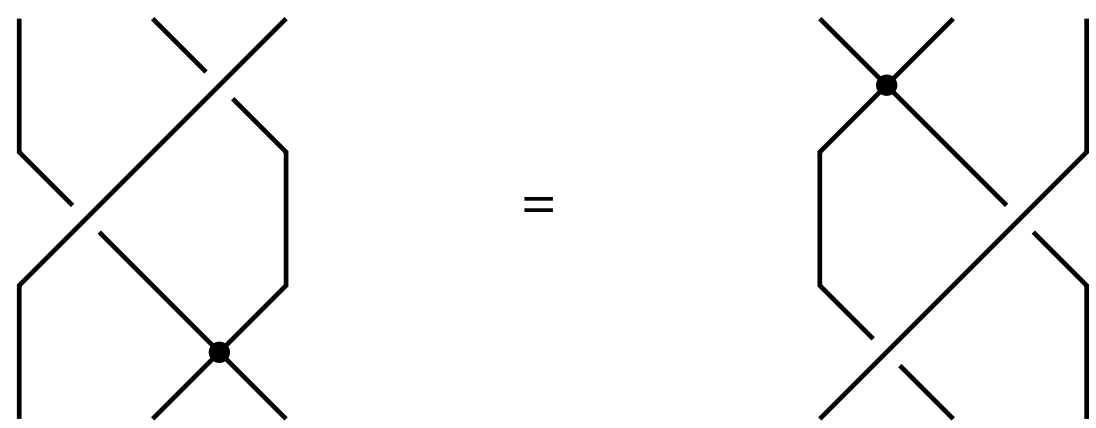

FIGURE 7

Proposition 9. For the fundamental word $\delta$, we have the following commutation formulas:

$$
\left\{\begin{array}{l}
a_{t s} \delta \doteq \delta a_{(t+1)(s+1)} \quad \text { for } 1 \leq r<s<t<n \\
a_{n s} \delta \doteq \delta a_{(s+1) 1}, \\
b_{t s} \delta \doteq \delta b_{(t+1)(s+1)} \quad \text { for } \quad 1 \leq r<s<t<n \\
b_{n s} \delta \doteq \delta b_{(s+1) 1}
\end{array}\right.
$$

Proof. For the generators $a_{t s}$, this was proved in [8]. Suppose that $1 \leq r<s<t<n$. Then, using relations (9), we obtain

$$
\begin{aligned}
b_{t s} \delta & \doteq b_{t s} a_{t s} a_{n(n-1)} a_{(n-1)(n-2)} \ldots a_{(t+1) s} a_{t(t-1)} \ldots a_{(s+2)(s+1)} a_{s(s-1)} \ldots a_{21} \\
& \doteq a_{t s} b_{t s} a_{n(n-1)} a_{(n-1)(n-2)} \ldots a_{(t+1) s} a_{t(t-1)} \ldots a_{(s+2)(s+1)} a_{s(s-1)} \ldots a_{21} \\
& \doteq a_{t s} a_{n(n-1)} a_{(n-1)(n-2)} \ldots b_{t s} a_{(t+1) s} a_{t(t-1)} \ldots a_{(s+2)(s+1)} a_{s(s-1)} \ldots a_{21} \\
& \doteq a_{t s} a_{n(n-1)} a_{(n-1)(n-2)} \ldots a_{(t+1) s} b_{(t+1) t} a_{t(t-1)} \ldots a_{(s+2)(s+1)} a_{s(s-1)} \ldots a_{21} \\
& \doteq a_{t s} a_{n(n-1)} a_{(n-1)(n-2)} \ldots a_{(t+1) s} a_{t(t-1)} b_{(t+1)(t-1)} \ldots a_{(s+2)(s+1)} a_{s(s-1)} \ldots a_{21} \\
& \doteq a_{t s} a_{n(n-1)} a_{(n-1)(n-2)} \ldots a_{(t+1) s} a_{t(t-1)} \ldots b_{(t+1)(s+2)} a_{(s+2)(s+1)} a_{s(s-1)} \ldots a_{21} \\
& \doteq a_{t s} a_{n(n-1)} a_{(n-1)(n-2)} \ldots a_{(t+1) s} a_{t(t-1)} \ldots a_{(s+2)(s+1)} b_{(t+1)(s+1)} a_{s(s-1)} \ldots a_{21} \\
& \doteq a_{t s} a_{n(n-1)} a_{(n-1)(n-2)} \ldots a_{(t+1) s} a_{t(t-1)} \ldots a_{(s+2)(s+1)} a_{s(s-1)} \ldots a_{21} b_{(t+1)(s+1)} \\
& \doteq \delta b_{(t+1)(s+1)} .
\end{aligned}
$$

For $t=n$, we have

$$
\begin{aligned}
b_{n s} \delta & \doteq b_{n s} a_{n(n-1)} a_{(n-1)(n-2)} \ldots a_{21} \doteq a_{n(n-1)} b_{(n-1) s} a_{(n-1)(n-2)} \ldots a_{21} \\
& \doteq \ldots \\
& \doteq a_{(n-1) n} a_{(n-1)(n-2)} \ldots a_{(s+2)(s+1)} b_{(s+1) s} a_{(s+1) s} \ldots a_{21} \\
& \doteq a_{(n-1) n} a_{(n-1)(n-2)} \ldots a_{(s+1) s} b_{(s+1) s} a_{s(s-1)} \ldots a_{21} \\
& \doteq a_{(n-1) n} a_{(n-1)(n-2)} \ldots a_{s(s-1)} b_{(s+1)(s-1)} a_{(s-1)(s-2)} \ldots a_{21} \\
& \doteq a_{(n-1) n} a_{(n-1)(n-2)} \ldots a_{s(s-1)} a_{(s-1)(s-2)} \ldots a_{21} b_{(s+1) 1}
\end{aligned}
$$

Geometrically, this commutation is shown in Figure 7

Analogs of other results proved by Birman, Ko, and Lee (which are also analogs of the statements of $\S \S 2$ and 3 of the present paper) remain valid for the singular braid monoid. They were proved by Chaynikov in 9 . 


\section{REFERENCES}

[1] S. I. Adyan, Fragments of the word $\Delta$ in the braid group, Mat. Zametki 36 (1984), no. 1, 25-34; English transl., Math. Notes 36 (1984), no. 1-2, 505-510. MR0757642 (86b:20048)

[2] A. I. Mal'tsev, On including of associative systems in groups. I, Mat. Sb. 6 (1939), no. 2, 331-336. (Russian) MR0002152 (2:7d)

[3] _ On including of associative systems in groups. II, Mat. Sb. 8 (1940), no. 2, 251-264. (Russian) MR0002895 (2:128b)

[4] P. S. Novikov, On the algorithmic unsolvability of the word problem in group theory, Trudy Mat. Inst. Steklov. 44 (1955), 3-143. (Russian) MR0075197(17:706b)

[5] E. Artin, Theorie der Zöpfe, Abh. Math. Semin. Univ. Hamburg 4 (1925), 47-72.

[6] J. C. Baez, Link invariants of finite type and perturbation theory, Lett. Math. Phys. 26 (1992), no. 1, 43-51. MR.1193625 (93k:57006)

[7] J. S. Birman, New points of view in knot theory, Bull. Amer. Math. Soc. (N.S.) 28 (1993), no. 2, 253-287. MR.1191478 (94b:57007)

[8] J. S. Birman, K. H. Ko, and S. J. Lee, A new approach to the word and conjugacy problems in the braid groups, Adv. Math. 139 (1998), no. 2, 322-353. MR1654165 (99m:20082)

[9] V. V. Chaynikov, Garside structure for singular braid monoid in Birman, Ko, Lee generators, Comm. Algebra 34 (2006), no. 6, 1981-1995. MR2235075 (2007c:20092)

[10] R. Corran, A normal form for a class of monoids including the singular braid monoids, J. Algebra 223 (2000), no. 1, 256-282. MR 1738262 (2001f:20123)

[11] O. T. Dasbach and B. Gemein, The word problem for the singular braid monoid, Arxiv.math. GT/9809070.

[12] E. El-Rifai and H. R. Morton, Algorithms for positive braids, Quart. J. Math. Oxford Ser. (2) 45 (1994), no. 180, 479-497. MR1315459 (96b:20052)

[13] D. B. A. Epstein, J. W. Cannon, D. F. Holt, S. V. F. Levy, M. S. Paterson, and W. P. Thurston, Word processing in groups, Jones and Bartlett Publishers, Boston, MA, 1992. MR.1161694 (93i:20036)

[14] R. Fenn, D. Rolfsen, and J. Zhu, Centralisers in the braid group and singular braid monoid, Enseign. Math. (2) 42 (1996), no. 1-2, 75-96. MR 1395042 (97h:20047)

[15] F. A. Garside, The braid group and other groups, Quart. J. Math. Oxford Ser. (2) 20 (1969), 235-254. MR 0248801 (40:2051)

[16] A. Járai, Jr., On the monoid of singular braids, Topology Appl. 96 (1999), no. 2, 109-119. MR 1702305 (2000f:57013)

[17] M. S. Putcha, Conjugacy classes and nilpotent variety of a reductive monoid, Canad. J. Math. 50 (1998), no. 4, 829-844. MR.1638627 (99f:20095)

Département des Sciences Mathématiques, Université Montpellier II, Place Eugéne BatailLON, 34095 Montpellier Cedex 5, France

E-mail address: vershini@math.univ-montp2.fr

Current address: Sobolev Institute of Mathematics, Novosibirsk 630090, Russia

E-mail address: versh@math.nsc.ru

Received 2/SEP/2008

Translated by THE AUTHOR 